\title{
The Kanehira-Hatusima 1940 Collection of New Guinea Plants. XIII.
}

\author{
By
}

\section{S. Hatusima}

Received May 5, 1942.

\section{Sparganium sp.}

\section{S. Hatusima : Sparganiaceae.}

No. 13766 Kanehira-Hatusima, Angi, Arfak Mts., April 8, 1940. Submersed in open marsh by Lake Giji at about $2,000 \mathrm{~m}$. altitude ; a herb, 30-50 $\mathrm{cm}$. high, shoots stoloniferous.

Unfortunately our material is sterile, but I have no doubt about its generic identification. No representative of this family is hitherto known from New Guinea.

\section{S. Hatusima : Flagellariaceae.}

Flagellaria indica Linn. Sp. Pl. (1753) 333; GibBs, Contr. Phytog. \& Fl. Arfak Mts. (1917) 202 ; Rendle in Dr. H. O. Forbes, New Guinea Plants (1923) 57 ; Krause in Nova Guinea 14 (1924) 180 et Engl. Bot. Jahrb. 59 (1925) 544.

Nos. 11636, 12885 Kanehira-Hatusima, Nabire, March 16, 1940; in rain-forests at $10 \mathrm{~m}$. altitude. Nos. 12221, 12244 Kanehira-Hatusima, Dallmann, $50 \mathrm{~km}$. inward of Nabire, March 3, 1940 ; scandent in Agathisforests at about $500 \sim 600 \mathrm{~m}$. altitude.

var. gracilis Hatusima, var. nov.

A typo recedit foliis angustioribus circ. $6 \mathrm{~mm}$. latis ad $22 \mathrm{~cm}$. longis, margine infra medium crispulatis, inflorescentiae minores.

No. 12004 Kanehira-Hatusima, Dallmann, March 1, 1940. Scandent in Agathis-forests at about $500 \mathrm{~m}$. altitude.

Distrib. Species Tropical Africa and Asia to Polynesia and northern Australia.

Hanguana malayana ( $\mathrm{J}_{\mathrm{ACK}}$ ) Merr. in Philip. Journ. Sci. 10 (1915) Bot. 3, Straits Branch Roy. Asiat. Soc. (1921) 109; KraUse in Nova Guinea 14 (1924) 180 et EngL. Bot. Jahrb. 59 (1925) 546.

Susum malayanum Planch. ex Hook. f. Fl. Brit. Ind. 6 (1892) 391 ; Lautb. in EngL. 1. c. 50 (1913) 289.

Veratrum? malayana JACE in Malay Misc. 1 (1820) 25. 
No. 12180 Kanehira-Hatusima, Dallmann, March 2, 1940. In Agathisforests at about $600 \mathrm{~m}$. altitude; very rare.

Distrib. Malaya, Philippines to Micronesia.

\section{S. Hatusima: Centrolepidaceae.}

Centrolepis novo-guineensis GibBs, Contr. Phytog. \& Fl. Arfak Mts. (1917) 99 .

No. 13645 Kanehira-Hanehira, Angi, Arfak Mts., April 6, 1940. In marsh on the summit of Mt. Koebre at about $2,300 \mathrm{~m}$. altitude. This is also fairly common in open sandy marsh by the Lake Gita at about $1,900 \mathrm{~m}$. altitude.

Distrib. Endemic; the type was from Angi.

\section{S. Hatusima : Xyridaceae.}

Xyris novo-guineensis Hatusima, sp. nov.

Xyris pauciflora (non Willd.) sensu GiBBs, l. c. 100.

Perennis, radicibus crassiusculis. Folia satis rigida, 10-20 cm. longa, circ. $2 \mathrm{~mm}$. lata, in sicco nervoso-striata; vagina quintam fere partem folii ocupans, superne lamina non latior, basi valde dilatata, inferne castanea nitidulaque. Scapi 50-60 cm. longi $1.5-2 \mathrm{~mm}$. crassi, subteretes nervosostriati glabri densissime flavescento-punctates, superine pluri-striati (cire. 8 ), inferne castanei nitidique. Spica pauciflora subglobosa circ. $1 \mathrm{~cm}$. lata et $0.8 \mathrm{~cm}$. alta ; bracteae chartaceae subnitidulae $3-4 \mathrm{~mm}$. longae infimae breves, obovato-rotundatae castaneae, apice emarginatae dorso carinatae 3-4 mm. longae, intermediae castaneae, ovato-ellipticae, 5-6 mm. longae, apice rotundatae, area dorsali nulla vulgo castanea. Sepala lateralia navicularia curvula circ. $5 \mathrm{~mm}$. longa, $3-3.5 \mathrm{~mm}$. lata, elliptico-spathulata, brunneo-flava, carina angusta integerrima, apice obtusa vix fimbriatolaciniata. Petala limbo obovato circ. $3 \mathrm{~mm}$. longo, basi cuneato; staminoidea bibrachiata, brachiis longe penicellatis ; antherae circ. $1.2 \mathrm{~mm}$. longae, 0.3 $\mathrm{mm}$. latae, connective angusto, filamentum antheris paullo breviore. Capsula pericarpio tenui, semina oblongo-ellipsoidea circ. $0.8 \mathrm{~mm}$. longa, $0.3 \mathrm{~mm}$. lata.

No. 13591 Kanehira-Hatusima, Angi, Arfak Mts., April 5, 1940. In open marsh by Lake Gita, at about 1,900 m. altitude.

This is most closely related to Xyris sumatrana MaLme from which it chiefly differs in its several ribs on the upper part of the stem, shorter leaves and much broader curved lateral sepals.

\section{S. Hatusima : Eriocaulacae.}

Eriocaulon leucogens RIDL. in Trans. Linn. Soc. ser. 2,. Bot. 9 (1916) 240; 
RENDLe in GibBs. l. c. 100.

No. 13542 Kanehira-Hatusima, Angi, Arfak Mts., April 5, 1940. In open marsh by Lake Gita at $2,000 \mathrm{~m}$. altitude.

Distrib. South-western New Guinea (Mt. Carstensz); endemic.

Aclisia macrophylla (R. BR.) Brückn. in Engler-PrantL, Nat. Pflanzenf. 2te Auf. 15 a (1930) 176.

Pollia macrophylla Benth. Fl. Austr. 7 (1878) 90; K. Schum. et Lautb. 1. c. 216 ; Lautb. in Engl. 1. c. 50 (1913) 58.

No. 11828 Kanehira-Hatusima, Nabire, Feb. 28, 1940. In high rainforests at about $300 \mathrm{~m}$. altitude. A herb, $60 \mathrm{~cm}$. high, fruits blue.

Distrib. Malaya, Philippines to northern Australia.

Aclisia Zollingeri Hassk. Commel. Ind. (1870) 51; BRüCKN. 1. c. 176.

Pollia Zollingeri C. B. Clanke in DC. Suit. Prodr. 3 (1881) 127 ; K. Schum. et Lautb. l. c. 216 ; Lautb. 1. c. 57.

No. 13002 Kanehira-Hatusima, Momi, March 23, 1940 . In dense rainforests. A herb, $80 \mathrm{~cm}$. high.

Distrib. Malaya to Formosa and China.

Aclisia sorzogonensis E. Mey. in Presl, Rel. Haenk. 1 (1825) 138, t. 25; BRÜCKN. l. c. 176.

Pollia sorzogonensis Engl. Gen. Pl. (1836-50) 1029; K. Schum. et Lautb. l. c. 216 ; Lautb. l. c. 57 ; Hallier in Nova Guinea 8 (1913) 907.

Nos. 11536, 11630 Kanehira-Hatusima, Nabire, Feb. 25, 1940. In dense rain-forests at $10 \mathrm{~m}$. altitude.

Distrib. India, Malaya to China.

\section{S. Hatusima: Commelinaceae.}

Aneilema papuana W ARB. in Engl. Bot. Jahrb. 13 (1891) 62; LaUtb. in Engl. ibid. 50 (1913) 62.

No. 11599 Kanehira-Hatusima, Nabire, Feb. 26, 1940; in high rainforests at about $3 \mathrm{~m}$. altitude. No. 13252 Kanehira-Hatusima, Momi, March 30, 1940; in high rain-forests.

Distrib. North-eastern New Guinea to Bismarck Archipelago.

Aneilema humile Warb. in Engl. l. c. 13 (1891) 270 ; K. Schum. et Lautb. Fl. Deutsch. Schutzg. Süds. (1901) 218; LaUtb. in ENGL. l. c. 50 (1913) 63.

No. 11536 Kanehira-Hatusima, Nabire, Feb. 25, 1940. In high rainforests.

Distrib. North-eastern New Guinea. 
Cyanotis capitata C. B. Clarke in DC. Suit. an Prodr. 3 (1881) 243; LAUTtB. 1. с. 65.

No. 13418 Kanehira-Hatusima, Momi, March 3, 1940. In high rainforests at $10 \mathrm{~m}$. altitude.

Distrib. India, China, Philippines to Malaya.

Cyanotis uniflora Hassk. Commel. Ind. (1870) 104 ; Lautb. l. c. 65.

No. 13381 Kanehira-Hatusima, Momi, April 3, 1940. In high rainforests at $10 \mathrm{~m}$. altitude.

Distrib. Malaya to Philippines.

Forrestia hispida Less. et A. Rich. Sert. Asterol (1834) 2, t. 1; Lautr. l. c. 64 ; Hallier 1. c. 906 (cum form.).

No. 13244 Kanehira-Hatusima, Momi, March 30, 1940. In edge of high rain-forests at $5 \mathrm{~m}$. altitude.

Distrib. Sumatra, Java and Formosa.

\section{S. Hatusima : Juncaceae.}

Juncus articulatis LinN. Sp. Pl. (1753) 327.

Juncus lampocarpus EнRH. Calam. Gram. et Tripetal. Excicc. n. 126. (1791); Rendle in Gibbs l. c. 100.

No. 13566 Kanehira-Hatusima, Angi, Arfak. Mts., April 5, 1940. In open marsh by Lake Gita at $1,900 \mathrm{~m}$. altitude.

Distrib. Eastern Asia, Europe to North Africa and northern America.

Juncus effusus var. decipiens Buchenau in ENGL. Bot. Jahrb. 12 (1890) 229, Juncac. (1906) 136.

Juncus effusus (non LinN.) sensu Diels in ENGL. 1. c. 62 (1929) 461,

Juncus decipiens NaKaI, Rep. Veg. Kamikoti' (1928) 35.

No. 13561 Kanehira-Hatusima, Angi, Arfak Mts., April 5, 1940. In open marsh by Lake Gita at 1,900 m. altitude.

Distrib. Widely distributed in Old World.

\section{S. Hatusima : Amarylidaceae.}

Curculigo latifolia Dryand var. villosa (Wall.) Baker in Journ. Limn. Soc. 17 (1875) 125; Ноок. f. Fl. Brit. Ind. 6 (1892) 280.

Curculigo villosa WaLl. Cat. n. 5763 A.

No. 12081 Kanehira-Hatusima, Dallmann, $45 \mathrm{~km}$. inward of Nabire, March 1, 1940. In edge of Agathis-forests at $500 \mathrm{~m}$. altitude.

Distrib. Malay Peninsula and Archipelago. A new addition to the flora of New Guinea. 
Curculigo recurvata AIt. Hort. Kew. ed. 2,2 (1811) 253; K. Schum. et Lautb. Fl. Deutsch. Schutzg. Süds. (1901) 222 ; Lautb. in Engl. 1. c. 50 (1913) 304.

Moliniera recurvata NeL in ENGL. l. c. 51 (1914) 8.

No. 11556 Kanehira-Hatusima, Nabire, Feb. 25, 1940. In secondary forests at $2 \mathrm{~m}$. altitude.

Distrib. India, China, Philippines to Malaya and northern Australia.

\section{S. Hatusima : Dioscoreaceae.}

Dioscorea bulbifera Linn. Sp. Pl. (1753) 1036; Kunth. in Engl. Pflanzenr. 87 Heft (IV. 43) (1924) 88; Kaneh. Enum. Micr. Pl. (1936) 291.

No. 11508 Kanehira-Hatusima, Nabire, Feb. 24, 1940. Seandent in edge of secondary rain-forests.

Distrib. India, China to Malaya.

Dioscorea ( $§$ Euantiophyllum, Salicifoliae) dallmannensis Hatusima, sp. nov., Fig. 1.

Caules laxe ramosi glabri circ. 1-1.5 mm. crassi. Folia alterna, lineari-oblonga, papyraceo-chartacea, apice acuminata longe angustata, basi levissime cordato-incisa, 3-nervia, utrinque glaberrima nitidula, in sicco utrinque pallide fuscescentia, 8$12 \mathrm{~cm}$. longa, $1-1.8 \mathrm{~cm}$. lata, nervis reticulatis supra paullo infra prominente elevatis; petiolo plerumque $2 \mathrm{~cm}$. longo glabro. Spicae in axillis foliorum vel in ramum terminalem efoliatum usque $15-20 \mathrm{~cm}$. longum 2- vel 3-natim dispositae, circ. $4-5 \mathrm{~cm}$. longae, 3 $\mathrm{mm}$. latae. Flores in

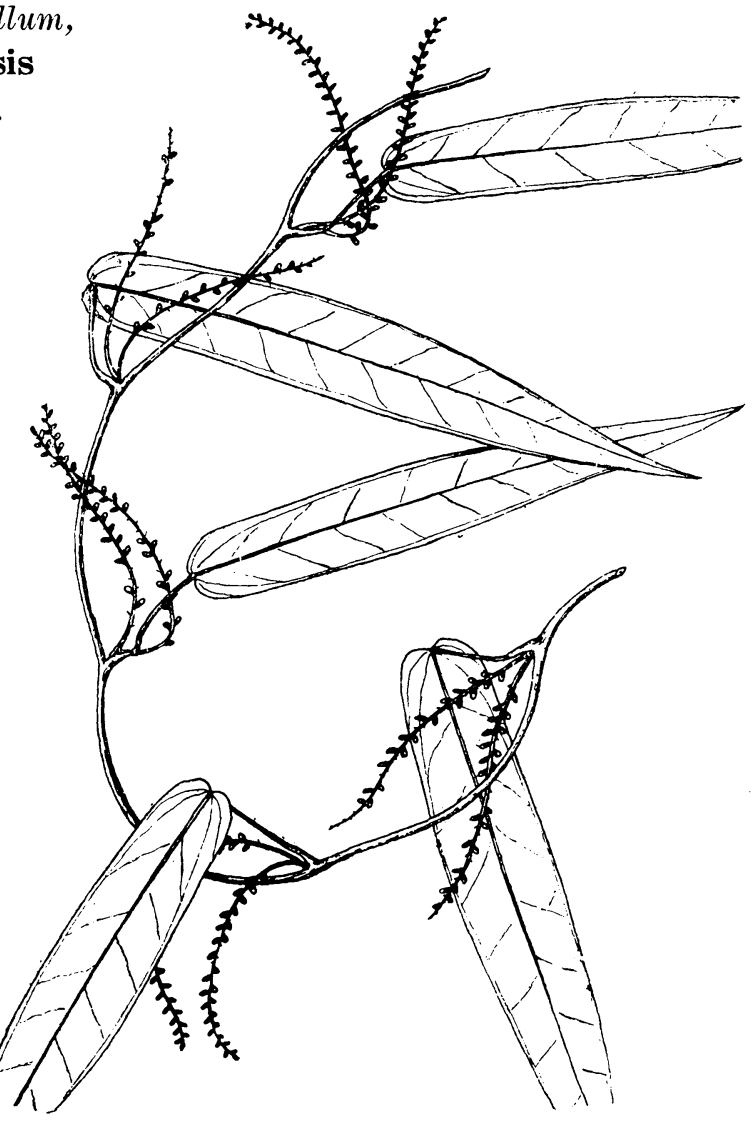

Fig. 1. Dioscorea dallmannensis Hatusima. 
spica numerosi (20-25) $1.2 \mathrm{~mm}$. remoti, sessiles, minuti vix $1 \mathrm{~mm}$. longi, bracteae anguste ovatae apice acuminatae circ. $1 \mathrm{~mm}$. longae. Perianthii segmenta exteriora obovato-spathulata, apice rotundata circ. $1.5 \mathrm{~mm}$. longa, interiora minora obovato-elliptica, revoluta vix $1 \mathrm{~mm}$. longa. Stamina fertilia 6 , inaequalia, exteriora brevissima, distica; interiora longiuscula, cum rudimento stylino usque ad medium filamentorum connata; antherae orbiculares circ. $0.5 \mathrm{~mm}$. longae.

No. 12301 Kanemira-Hatusima, Dallmann, Nabire, March 1, 1940. Scandent in edge of Agathis-forests at $500 \mathrm{~m}$. altitude.

This is most closely related to Dioscorea grata Prain et Burkill from the Philippines, from which it differs by its much longer spikes with somewhat larger male flowers.

Dioscorea nummularia Lam. Encycl. 3 (1789) 231; Kunth l. c. 282.

No. 14225 Kanehira-Hatusima, Waren, April 19, 1940. Scandent in edge of rain-forests at $2 \mathrm{~m}$. altitude.

$\dot{D}$ istrib. Philippines, Celebes to Micronesia and Bismarck Archipelago.

Dioscorea pentaphylla Linn. Sp. Pl. (1753) 1032; Kunth l. c. 145.

No. 13225 Kanehira-Hatuisma, Waren, March 26, 1940. Scandent in: edge of strand-forests.

Distrib. India through Malaya to Polynesia.

\section{Dioscorea sp.}

No. 14223 Kanehira-Hatusima, Momi, April 19, 1940. Scandent in edge of rain-forests at $3 \mathrm{~m}$. altitude.

This sterile collection is very closely related to Dioscorea Sarasinii ULINE, from which it differs by its somewhat narrower leaves with much sinuated bases.

\section{S. Hatusima : Iridaceae.}

Patersonia novo-guineensis Gibbs, l. c. 101; Went in Nova Guinea 14 (1924) 114.

Nos. 13691, 13663 Kanehira-Hatusima, Angi, Arfak Mts., April 6, 1940. Abundant in open summit of Mt. Koebre at about 2,300 m. altitude. Distrib. Endemic; the type was from Angi.

\section{S. Hatusima : Musaceae.}

Heliconia bihai Linn. Mant. 2 (1771) 211; K. Schum. et Lautb. l. c. 224. No. 13334 Kanehira-Hatusima, Momi, April 3, 1940. In edge of rainforests; a herb, $3 \mathrm{~m}$. high, flowers pale yellow. 
Distrib. Origin of America, now naturalized in Malaya and Polynesia.

\section{S. Hatusima: Maranthaceae.}

Cominsia gigantea (Scheff.) K. Schum. in Engl. Pflanzenr. (Marant.) (1902) 58 ; K. Schum. et Lautb. l. e. Nachtr. (1905) 68 ; Loesener in Engl.Prantl, Pflanzenf. 2te Aufl. Bd. 15 a (1930) 673, f. 302, G. H.

Phrynium giganteum Scheff. in Ann. Jard. Buitenz. 1 (1876) 58.

No. 11542 Kanehira-Hatusima, Nabire, Feb. 25, 1940; in high rainforests at $10 \mathrm{~m}$. altitude, a herb, $1 \mathrm{~m}$. high. No. 12430 Kanehira-Hatusima, Patema, $40 \mathrm{~km}$. inward of Nabire at about $400 \mathrm{~m}$. altitude, March 6, 1940 ; in rain-forests.

Distrib. Amboina.

Cominsia maxima Hatusima sp. nov., Fig. 2.

Herba perennis ad $5 \mathrm{~m}$. alta. Folia magna, ovato-oblonga, chartacea, ad $130 \mathrm{~cm}$. longa, $50 \mathrm{~cm}$. lata, apice mucronata, basi subtruncata, utrinque glabra, costa media utrinque elevata, basi $1 \mathrm{~cm}$. crassa, nervis lateralibus utrinsecus circ. 50, parallelis, a se 2-2.5 cm. remotis, secundariis numerosis a se $1.5 \mathrm{~mm}$. remotis, supra vix subtus prominente elevatis. Inforescentiae in caule foliato terminales, paniculatae, ad $60 \mathrm{~cm}$. longae, rhachis rhachillisque fuscescentia densiuscule fusco-hirsuta, rhachillis circ. $2-3 \mathrm{~mm}$. crassa, bracteis distichis, oblongo-lanceolatis, deciduis, apice acutis ad $4.5 \mathrm{~cm}$. longis, $1 \mathrm{~cm}$. latis, glabris, 2-4-floris, flores subsessiles; sepala 3 , anguste oblanceolata apice obtusa, $1.2 \mathrm{~cm}$. longa, ad $2 \mathrm{~mm}$. lata, membranacea; corollae tubus graciles ad $4.5 \mathrm{~cm}$. longae, $1 \mathrm{~mm}$. crassae, lobi valde irregulares, membranaceis, albi, laterales 2, naviculares margine cristati, $6-7 \mathrm{~mm}$. longi; labellum obovato-ellipticum apice obtusiusculum $1.3 \mathrm{~cm}$. longum, 7-8 mm. latum, intus medio in longitudinem cristis compressis, dolabratis circ. $3 \mathrm{~mm}$. longis et altis suffultum. Stamina incurva. Ovarium 3-loculare, ellipsoideum $3 \mathrm{~mm}$. longum $1.5 \mathrm{~mm}$. latum

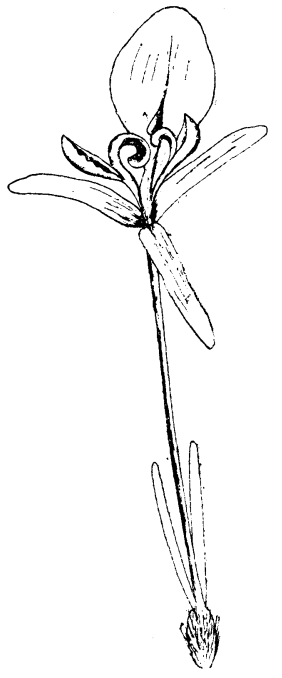

Fig. 2. Cominsia maxima Hats. (flower) villosum. Fructus circ. $1 \mathrm{~cm}$. longus, globosus, pericarpio crassiuscule glabro. Semina 3, oblonga angulata $7 \mathrm{~mm}$. longa $5 \mathrm{~mm}$. lata nigrescentia. No. 12983 Kanehira-Hatusima, Waren, March 23, 1940. In rainforests at $10 \mathrm{~m}$. altitude.

This is most closely related to Cominsia gigantea (SchefF.) K. Schum., from which it differs in its more robust status, much larger leaves and 
pubescent inflorescences bearing quite different flowers.

Donax canniformis (Forst. f.) K. Schum. in ENGL. Bot. Jahrb. 15 (1893) 440 ; Loesener l. c. 668 ; KaneH. l. c. 293.

Thalia canniformis Forst. f. Prodr. (1786) 1.

No. 11486 Kanehira-Hatusima, Nabire, Feb. 24, 1940. In high dilluvial rain-forests.

Distrib. From Java, Borneo and the Philippines eastward to Palau, Aru and Polynesia.

Phrynium capitatum Willd. Sp. Pl. 1 (1797) 17 ; Loesener l. c. 673, f. $302 \mathrm{E}$.

No. 11728 Kanehira-Hatusima, Nabire, Feb. 27, 1940; in high rainforests; a herb $1 \mathrm{~m}$. high, flowers red. No. 12407 Kanehira-Hatusima, Patema, March 6, 1940; in high rain-forests at about $400 \mathrm{~m}$. altitude.

Distrib. India to Central China. New to the flora of New Guinea.

Phrynium macrocephalum K. Schum. Fl. Kaiser Wilhelmsl. (1889) 30; Warb. in Engl. Bot. Jahrb. 13 (1891). 280; K. Schum. et Lautb. l. e. 237; LOESENER 1. c. 673.

Nos. 11450 (fr.), 11574 (fl.) Kanehira-Hatusima, Nabire, April 23, 1940. In dilluvial rain-forests at $10 \mathrm{~m}$. altitude; a herb, $1 \mathrm{~m}$. . in height, flowers white.

Distrib. North-eastern New Guinea.

\section{金平・初岛探集ニユーギニヤ植物研究 XIII（和交摘要)}

初島住彦

みくり科 本科ノ植物ハニユーギニヤデハ未記錄デアツタガ 今包アンギ湖ノ湖畔 二內地ノみくりニ近イ一種 發見スルコトガ出來夕。探集品八花モ, 果實モナイ標 本デ種ノ決定八困難デアルガみくり類ナルコトハ間違ナイ。涼洲ニハSparganium angustifolium L. ガ知ラレテキルガ Angi 地方座ノモノ八葉ガ大分廣ク同一物デ八 ナサ相デアル。

とううるもどき科 アジヤ/熱帶地方二普通ナとうつるるどきノ外, 基本種二比 シ葉ガ著シク狹ク葉緣/中部以下=鋸苳狀/突起アル一變種ヨダルマン地方デ發見 シタ。ミクロネシヤニモアル Hanguana malayana MERR. 八今回/探集地デ八極メ テ稀デダルマン地方ノAgathis 林內二筫弱ナモノョ 2〜3 株見受ケタ二過ギナカツ タ。 
Centrolepidaceae Centrolepis novo-guineensis GIBBS 本虽八涼洲系/植物デ他 デハニユーギニヤ, ホルネオ, 比島, 佛印二夫々一種宛產スル二過ギナイ。水邊二多 イ團塊植物ノ一種デ高サ一寸內外ノ植物デアル。

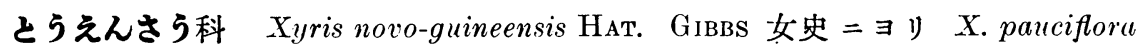
WILLD. 卜鑑定サレテキタモノデアルガ，スマトラ産，X. sumatrana MALME =最 モ近イ別種デアル。莖ノ縱稜ノ多イコト，葉ノ短イコト，側方蓦片ガ廣イノデスマ トラ座ト區別デキル。

ほしくさ科 Eriocaulon leucogens RIDL. 內地ノごましほほしくさヨ思八セル種類 デ生育地ニヨリ大为）變化ガ激シイコトハ內地產同樣デアル。

つゆくさ科 總テ低地林ノ植物デ廣分布ノ種類ガ多ク固有種ハ少イ。

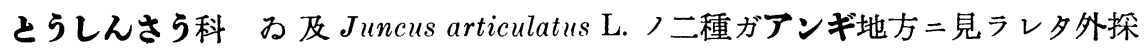
集ハシナカツタガ Luzula campaestris L. ヨ思ハセル sterile ノ植物アアンギ地方イ ライ河ノ河岸ノ岩石上二發見シ夕。

ひがんばな科 Curculigo recurvata AIT. 八低地林內二普通二見ラレ，印度方面， 產デ從來ニューギニヤニ八未記錄デアツタ C. latifolia var. villosa $\mathrm{BAK}$. ヨ今包ダル マン地方デ發見シタ。葉八長サ 4〜5 尺ニモナり裹面二白毛ガ密生シテキル

やまのいる科 本科八總テ低地林二限ラレ廣分布/種類ガ多イ。Dioscorea dallmanensis HAT. 八今包ダルマン地方デ發見シタ新種デ比島産, D. grata PRAIN et BURKILI 二最モ近イガ葉八幅狹ク，花序八長ク，雄芯ガ大キイノデ區別出來ル。

あやめ科 Patersonia novo-guineensis GIBBS 本屬八濠洲系ノ植物デニニーギニヤ 二二種, ボルネオ及比島二夫々一種宛知ラレテキル。本種八高サ 5 寸內外デ葉八硬 ク, 密二重リ合ツテ出ル。アンギ湖附近, 乾イ夕尾根, 原野二群生シテサル。

ばせう科 Heliconia hihai L. 南米原產デ現今デハアジヤ/熱帶地二野生化シテキ ル。高サ 2〜3 米二達シ一見みようが科ノ植物 思八セル。佾本科デハナビレ附近 ノ河岸林內二野生ノMusa sp. ヨ發見シタガ完全ナ標本ガナカツタノデ 鑑定不可能 デアツタ。

くずらこん科 本科ノ植物八通常冲積層上ノ低地林內二限ラレ美麗ナ花ヨ着ケル モノガ多イ。Cominsia maxima HAT. 入今包モミ附近ノ森林內デ發見シタ新種デ草 丈 5 米二モ達シ, ソノ大ナル葉八長サ 1.5〜2 米, 幅 50〜80 糎二モ達シ, ソレガ大キ ナ一本ノ葉柄ノ上二頂生シテキル樣八實二壯觀デアル。本科ノ植物中デハ恐ラク最 大ノモノデアラウ。一番近イノハ C. gigantia $\mathrm{K} . \mathrm{ScH}$. デアルガ葉八更二大キク, 花 序有毛ナル點デ容易二區別出來ル。 